\title{
Simultaneous measurements of strong-field ionization and high harmonic generation in aligned molecules
}

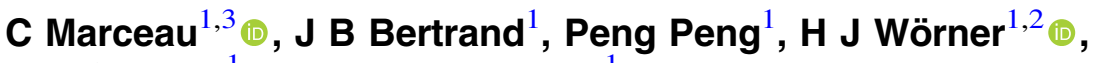 \\ P B Corkum ${ }^{1}$ and D M Villeneuve ${ }^{1}$ (1) \\ ${ }^{1}$ Joint Attosecond Science Laboratory, National Research Council and University of Ottawa, 100 Sussex \\ Drive, Ottawa, ON K1A 0R6, Canada \\ ${ }^{2}$ Laboratorium für physikalische Chemie, ETH Zürich, Wolfgang-Pauli-Strasse 10, 8093 Zürich, \\ Switzerland
}

E-mail: claude.marceau.2@gmail.com

Received 27 December 2019, revised 24 January 2020

Accepted for publication 13 February 2020

Published 25 March 2020

\begin{abstract}
High harmonic spectroscopy relies on high harmonic generation (HHG) in aligned molecules. The first step of HHG is the ionization of the molecule in the intense femtosecond laser field. Here we present measurements of both ionization yield and high harmonic yield as a function of molecular angle in $\mathrm{N}_{2}$ and $\mathrm{CO}_{2}$ molecules. Measurements were done at two wavelengths, 800 and $1200 \mathrm{~nm}$, and for a range of laser intensities, to study the sensitivity of laser conditions on both processes. The behavior of $\mathrm{N}_{2}$ was relatively insensitive to laser conditions. However in $\mathrm{CO}_{2}$, a minimum in high harmonic emission was observed that was sensitive to both laser intensity and wavelength, and was attributed to interference in emission from the HOMO and HOMO-2 orbitals.
\end{abstract}

Keywords: ionization yield, high harmonic generation, aligned molecules

(Some figures may appear in colour only in the online journal)

\section{Introduction}

High harmonic generation ( $\mathrm{HHG}$ ) has proven to be a sensitive probe of electronic structure in molecules, allowing angströmsize reconstruction of molecular orbitals [1-3]. It holds the promise of probing the instantaneous electronic structure of a molecule during a chemical reaction [4]. Up to date, a broad range of time-resolved experiments have confirmed the sensitivity of HHG to molecular dynamics [5] such as: rotation $[6,7]$, vibration [8,9], bound [10] and chemically-induced unbound nuclear motion [11]. Another class of dynamics, electron-hole dynamics [12, 13], resulting from the participation of multiple electronic states has been inferred from high-harmonic spectra in aligned molecules [14, 15].

The HHG process can be understood in the formalism of the three-step recollision model $[16,17]$. In step 1 , an electron is

3 Author to whom any correspondence should be addressed. tunnel-ionized from a given bound electronic state, in step 2, it is first accelerated away then back towards its parent ion to, finally, in step 3, photorecombine back to its ground state via a dipolar transition. When multiple electronic states contribute to HHG, the coherent sum of their high-harmonic emission leads to an interference controllable by molecular alignment and the driving laser field parameters, such as the peak intensity $[14,18]$ and wavelength [19]. The first step of HHG is the strong-field ionization (SFI) of the molecule. This step depends on the orientation of the molecule relative to the polarization direction of the laser. Depending on the orientation, different orbitals might be active.

SFI of atoms has been extensively modeled, based on the Ammosov-Delone-Krainov (ADK) model [20-22]. This atomic model has been developed further [23], and was generalized to molecules in a technique called molecular ADK, or MO-ADK [24, 25].

In this paper, we present the first simultaneous measurements of SFI and high-harmonic yield as a function of the 
molecular alignment in aligned molecules: $\mathrm{N}_{2}$ and $\mathrm{CO}_{2}$. Keeping the alignment conditions unchanged, we systematically survey the effect of changing the driving field wavelength ( 800 and $1200 \mathrm{~nm})$ and intensity $\left(I_{\text {probe }}=0.8-2.3 \times 10^{14} \mathrm{~W} \mathrm{~cm}^{-2}\right)$.

We observe that, at $800 \mathrm{~nm}$ probe wavelength, $\mathrm{HHG}$ and SFI strongly depend on intensity for both $\mathrm{N}_{2}$ and $\mathrm{CO}_{2}$. In $\mathrm{N}_{2}$, both ionization and HHG always peak when molecules are aligned parallel to the driving field $\left(\alpha=0^{\circ}\right)$. When the intensity of the latter is increased, the angular modulation becomes slightly weaker for the harmonic yield and significantly less pronounced for the molecular frame (MF) ionization. We observe the onset of ionization saturation around $I_{\text {probe }}=2.0 \times 10^{14} \mathrm{~W} \mathrm{~cm}^{-2}$. While the maximum angular modulation of the high-harmonic signal always occurs in the cutoff spectral region at $800 \mathrm{~nm}$, independently of the laser intensity, it rather always occurs around $40 \mathrm{eV}$ at $1200 \mathrm{~nm}$ probe wavelength. These measurements of HHG in aligned $\mathrm{N}_{2}$ at longer wavelength (than $800 \mathrm{~nm}$ ) provide evidence for dynamical effects that take place, along the lines of multiple orbitals ionization-induced hole dynamics [26].

In $\mathrm{CO}_{2}$, we follow the position of the spectral minimum at both 800 and $1200 \mathrm{~nm}$ and see intensity-dependent structures which were not reported earlier [14]. In addition, the simultaneous measurement of SFI allows us to observe the onset of a sharp MF angular ionization profile peaking around $42^{\circ}$ as the laser intensity is decreased. This trend qualitatively agrees with previous experimental results on SFI in $\mathrm{CO}_{2}$ [27].

\section{Experimental results and discussion}

\subsection{Impulsive molecular alignment}

A Ti:Sapphire multi-pass laser system $(32 \mathrm{fs}, 800 \mathrm{~nm}, 50 \mathrm{~Hz}$, $12 \mathrm{~mJ}$ per pulse) was used to perform all measurements. The molecules were first impulsively aligned [28, 29] using a stretched non-ionizing pump pulse $(70 \pm 5 \mathrm{fs}, 800 \mathrm{~nm}$, $I_{\text {align }}=5 \times 10^{13} \mathrm{~W} \mathrm{~cm}^{-2}$ ). After a delay time, the molecules were probed with a more intense laser pulse $(800 \mathrm{~nm}$ : $32 \pm 2$ fs or $1200 \mathrm{~nm}: 40 \pm 5$ fs of variable intensity). The probe pulse served to ionize the molecules, and at the same time to create an XUV spectrum by HHG. In addition to the $800 \mathrm{~nm}$ pulses from the Ti:Sa laser system, $1200 \mathrm{~nm}$ pulses $(\sim 1 \mathrm{~mJ})$ were generated by a high-energy optical parametric amplifier (HE-TOPAS) seeded by a fraction of the laser output $(\sim 8 \mathrm{~mJ})$. The intensity was adjusted with neutral density filters in both pump and probe arms of the MachZehnder interferometer. The polarization of the pump pulse was controlled by a half-wave plate.

The linearly polarized pump and probe beams were focused $(f=50 \mathrm{~cm})$ into the supersonic gas jet emanating from a pulsed valve (Parker) that introduced the analyte molecule $\left(\mathrm{N}_{2}\right.$ or $\left.\mathrm{CO}_{2}\right)$. The laser pulses were focused $\sim 2 \mathrm{~mm}$ before the gas target to select the HHG short trajectories [30], and $\sim 1 \mathrm{~mm}$ away from the pulsed valve orifice $(250 \mu \mathrm{m}$ diameter) in order to obtain cooler molecules. A negatively biased wire mesh located $10 \mathrm{~cm}$ from the nozzle orifice recorded the production of cations, which is a measure of the volume-integrated ionization probability. The linearity of this ion detector was verified [31]. The HHG spectra were recorded by an extreme ultraviolet (XUV) spectrometer composed of a concave XUV flatfield grating, a microchannel plate detector backed by a phosphor screen and a camera readout.

In figure 1, we present the time evolution of rotational wave packets as probed by high-harmonic and ionization yield measurements in (b) $\mathrm{N}_{2}$ and (c) $\mathrm{CO}_{2}$ molecules around their respective half-revival times. The high harmonic yields are spectrally integrated over a single harmonic order. The polarization directions of the aligning $\left(I_{\text {align }}=5 \times 10^{13} \mathrm{~W} \mathrm{~cm}^{-2}\right)$ and probing $\left(I_{\text {probe }}=1.5 \times 10^{14} \mathrm{~W} \mathrm{~cm}^{-2}\right)$ pulses are parallel. In $\mathrm{N}_{2}\left(\mathrm{CO}_{2}\right)$, the maximum degree of alignment is achieved at $\sim 4.12$ ps $(\sim 21.15 \mathrm{ps}$ ) pump-probe delay corresponding to half-revivals $[6,7,32]$. When alignment occurs, the molecular internuclear axis distribution is prolate (cigar shaped) along the pump pulse polarization. Shortly following is anti-alignment (see figure 1(b) and (c)) where the alignment distribution becomes oblate [32] (pancake shaped). At maximum alignment, for both $\mathrm{N}_{2}$ and $\mathrm{CO}_{2}$, our experimental conditions $\left(P_{\text {back }}=2\right.$ atm., $T_{\text {rot }}=30-40 \mathrm{~K}, I_{\text {align }}=5 \times 10^{13} \mathrm{~W} \mathrm{~cm}^{-2}$ and $\tau_{\text {align }}=70 \pm 5 \mathrm{fs}$ ) suggest a $3 \mathrm{D}$ degree of alignment of $\left\langle\cos ^{2} \theta^{\prime}\right\rangle=0.60 \pm 0.05$ based on calculations [33] and supported by supersonic gas expansion studies in similar conditions [34].

In $\mathrm{N}_{2}$ (figure 1(b)), both the high-harmonic and ionization yields maximize when molecules are aligned parallel to the probing field (delay $\sim 4.12 \mathrm{ps}$ ). The ratio of signal aligned:anti-aligned in ionization is around 1.5:1 and increases monotonically with high-harmonic order, exceeding 10:1 for cutoff harmonics.

In $\mathrm{CO}_{2}$ (figure $1(\mathrm{c})$ ), in contrast to the case of $\mathrm{N}_{2}$, the high-harmonic yield generally anti-correlates with ionization. This anti-correlation, reported earlier in [7], suggested evidence for two-center destructive quantum interference taking place at the recombination step in aligned $\mathrm{CO}_{2}$ molecules [35]. However, the presence of a dynamical (laser-dependent) local maximum in high-harmonic yield at alignment in the cutoff region (see H33 in figure 1(c)) is due to the dynamical interplay of multiple orbitals [14, 19].

We now proceed to study the dependence of both ionization and high-harmonic yields as a function of alignment angle $\alpha$ between the alignment and HHG probe polarization axes. For this, in aligned $\mathrm{N}_{2}\left(\mathrm{CO}_{2}\right)$, we set the pumpprobe delay to $\sim 4.12 \mathrm{ps}(\sim 21.15 \mathrm{ps})$ and rotate the polarization axis of the pump pulse with a half wave plate.

\subsection{Strong field ionization in aligned molecules}

The ionization signal measured in the laboratory frame (LF) $S(\alpha)$, is a convolution of the MF ionization profile $I(\theta)$ with the prolate molecular internuclear axis distribution $P\left(\theta^{\prime}, \phi^{\prime}\right)$. Here $\theta$ is the angle between the laser field polarization and the molecule's internuclear axis. The geometry is shown in 
(a)

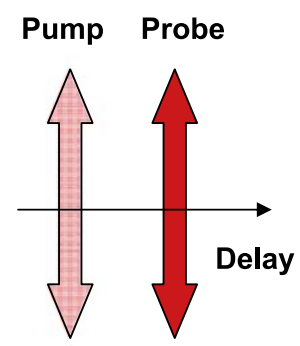

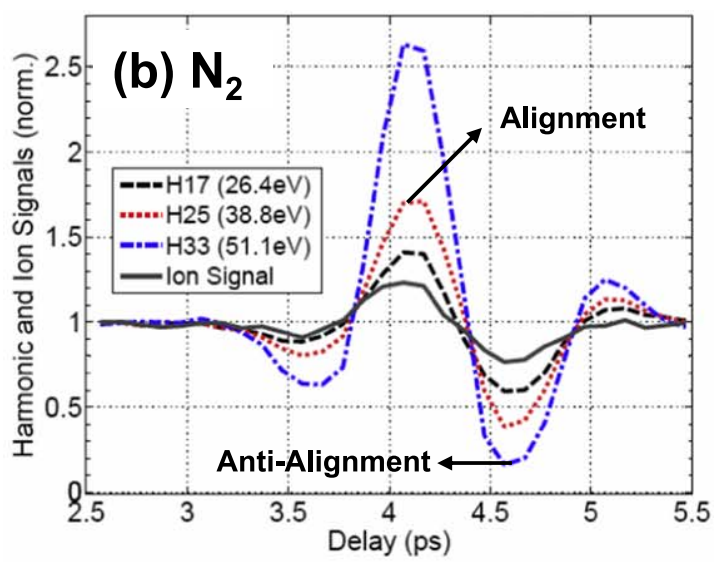

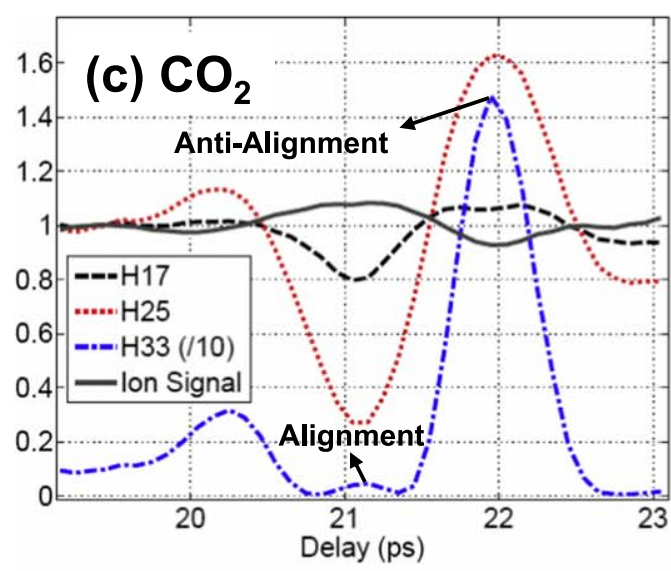

Figure 1. Rotational wavepackets are created via impulsive alignment with an $800 \mathrm{~nm}$ laser pulse. The molecules periodically rephase at revival times that depend on the molecule $\left(8.4 \mathrm{ps}\right.$ for $\mathrm{N}_{2}$ and $42.7 \mathrm{ps}$ for $\left.\mathrm{CO}_{2}\right)$. Here the molecules are probed around the half-revival times with an intense $800 \mathrm{~nm}$ laser pulse with an intensity of $1.5 \times 10^{14} \mathrm{~W} \mathrm{~cm}^{-2}$. (a) Shows that parallel polarizations were used for the pump and probe pulses. The results for ionization yield and high-order harmonics are shown for (b) $\mathrm{N}_{2}$ and (c) $\mathrm{CO}_{2}$. At the time of maximum alignment (shown by arrows), the molecular internuclear axis distribution is prolate along the pump pulse polarization and has a 3D degree of alignment estimated to be $\left\langle\cos ^{2} \theta^{\prime}\right\rangle=0.60 \pm 0.05$ for our experimental conditions. At the time of anti-alignment, the molecular axes are mostly aligned perpendicular to the laser polarization.

\section{ionizing field}

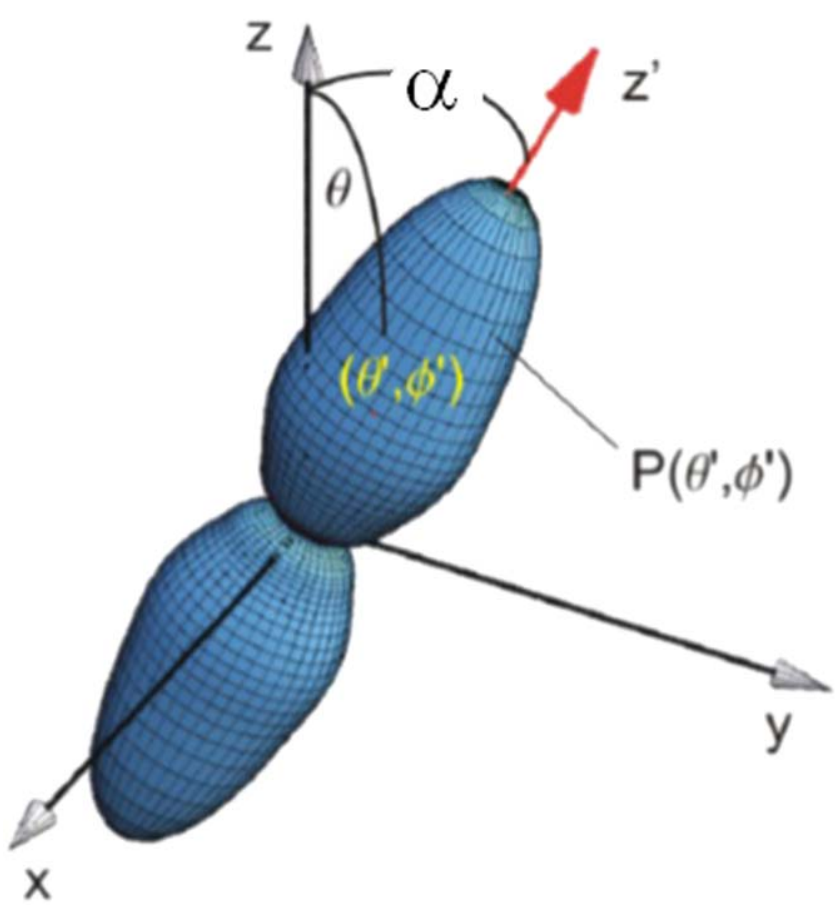

Figure 2. Definition of the molecular frame of reference. The distribution of molecular angles in the laboratory frame is given by $P\left(\theta^{\prime}, \phi^{\prime}\right)$. The angle of an individual molecule to the laboratory $Z$ axis is $\theta$.

figure 2. In integral form

$S(\alpha)=\int_{\theta^{\prime}=0}^{\theta^{\prime}=\pi} \int_{\phi^{\prime}=0}^{\phi^{\prime}=2 \pi} I\left(\theta\left(\theta^{\prime}, \phi^{\prime}, \alpha\right)\right) P\left(\theta^{\prime}, \phi^{\prime}\right) \sin \theta^{\prime} \mathrm{d} \phi^{\prime} \mathrm{d} \theta^{\prime}$ $\theta\left(\theta^{\prime}, \phi^{\prime}, \alpha\right)$ is defined by the LF to MF coordinate transformation [27]

$$
\cos \theta=\cos \alpha \cos \theta^{\prime}-\sin \alpha \sin \theta^{\prime} \sin \phi^{\prime}
$$

The experimental ionization yield $S(\alpha)$ is recorded as a function of the angle $\alpha$ between the aligning pulse polarization and that of the probe pulse. We wish to determine the molecularframe ionization probability $I(\theta)$, which is convolved with the alignment distribution $P\left(\theta^{\prime}, \phi^{\prime}\right)$ via equation (1). We parameterize $I(\theta)$ using the first 4 even normalized Legendre polynomials $l_{i}(\theta): I(\theta)=\left|\sum_{i=0}^{3} a_{2 i} l_{2 i}(\theta)\right|$. We parametrize the molecular axis distribution $P\left(\theta^{\prime}, \phi^{\prime}\right)$ following [27], using $\left\langle\cos ^{2} \theta\right\rangle=$ $0.55,0.6,0.65$ to cover the uncertainty in the degree of alignment. A least-squared optimization procedure is used to determine the MF ionization probability $I(\theta)$.

Figure 3 shows the LF ionization of $\mathrm{N}_{2}$ (scatter) using a $800 \mathrm{~nm}$ probe pulse at three peak intensities. The deconvoluted MF ionization probability distributions $I(\theta)$ of $\mathrm{N}_{2}$ are shown in green using $\left\langle\cos ^{2} \theta^{\prime}\right\rangle=0.55$ (dashed), 0.60 (solid) and 0.65 (dotted). With a $800 \mathrm{~nm}$ probe and $I_{\text {probe }}=1.0 \times$ $10^{14} \mathrm{~W} \mathrm{~cm}^{-2}$, the $\sim 3: 1$ ratio between parallel to perpendicular alignment is consistent with previous studies and reflects the $\sigma_{g}$ symmetry of the highest occupied molecular orbital (HOMO) of $\mathrm{N}_{2}$ [27, 36-38]. The small local maximum of ionization featured at $\theta=90^{\circ}$ is real as it was also reported elsewhere at lower intensity $\left(I_{\text {probe }}=3.0 \times 10^{13} \mathrm{~W} \mathrm{~cm}^{-2}\right)$ via COLTRIMS measurements [39]. As the intensity of the ionizing pulse increases, the parallel:perpendicular ratio decreases and the angular profile becomes almost isotropic. This is likely due to saturation of the ionization probability, which smooths out the angular dependence of ionization.

Figure 4 shows the same measurements in $\mathrm{CO}_{2}$. In both $\mathrm{N}_{2}$ and $\mathrm{CO}_{2}$, more sharply peaked features in the $\mathrm{MF}$ 

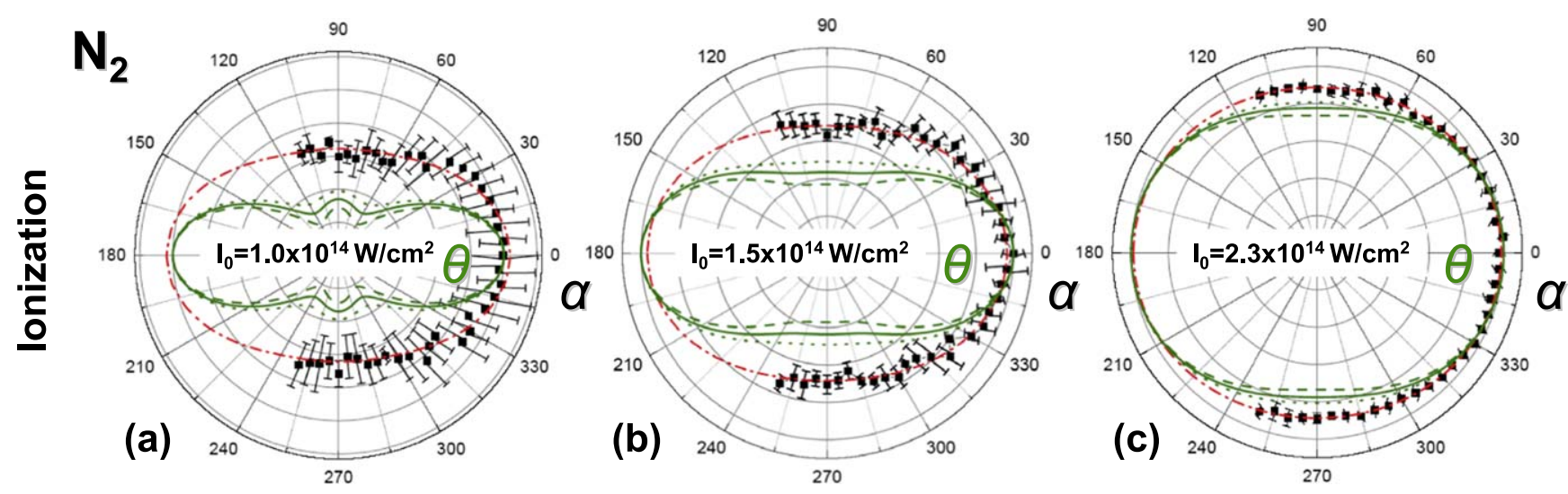

Figure 3. $\mathrm{N}_{2}$ ionization signal versus alignment angle $\alpha$ with $\lambda=800 \mathrm{~nm}$ for different intensities of the driving field. $I_{\text {probe }}=1.0$ (a), 1.5 (b) and $2.3(\mathrm{c}) \times 10^{14} \mathrm{~W} \mathrm{~cm}^{-2}$. From the laboratory frame ionization signal (scatter) and its Legendre polynomial fit (red dashed-dotted line), we retrieved the MF ionization profile $I(\theta)$ using the degree of alignment $\left\langle\cos ^{2} \theta^{\prime}\right\rangle=0.55$ (dash), 0.60 (solid) and 0.65 (dotted).
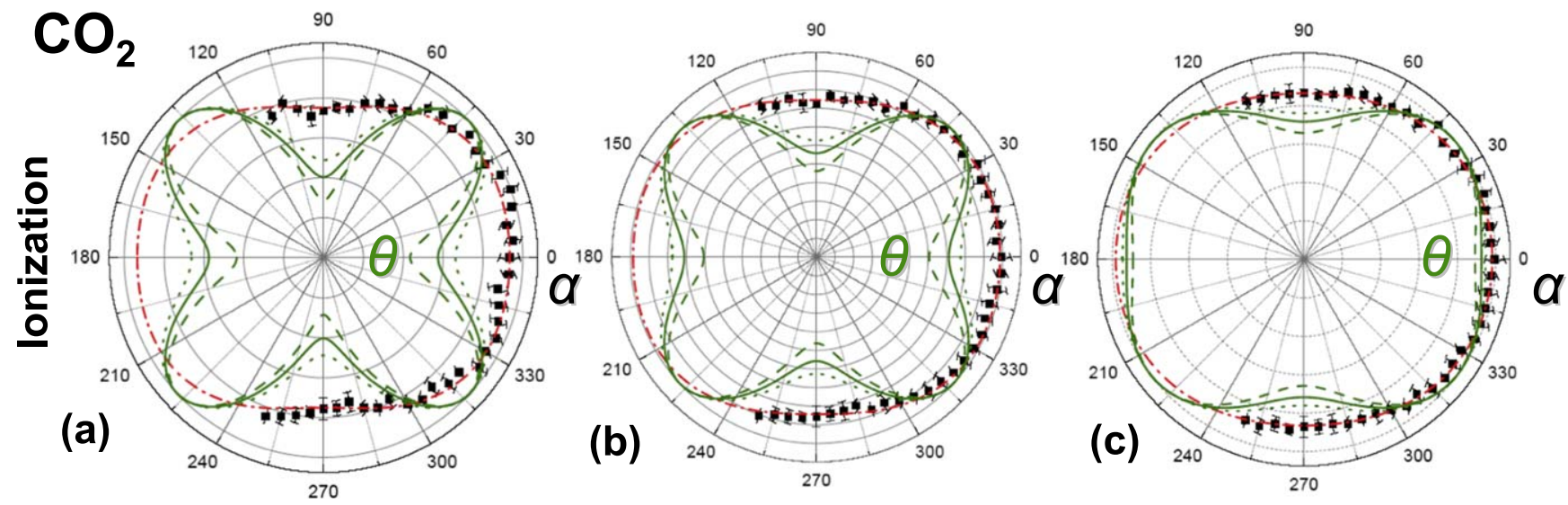

Figure 4. $\mathrm{CO}_{2}$ ionization signal versus alignment angle $\alpha$ with $\lambda=800 \mathrm{~nm}$ for different intensities of the driving field. $I_{\text {probe }}=1.0$ (a), 1.5 (b) and $2.3(\mathrm{c}) \times 10^{14} \mathrm{~W} \mathrm{~cm}^{-2}$. As in figure 3, we retrieved the MF ionization profile $I(\theta)$ using the degree of alignment $\left\langle\cos ^{2} \theta^{\prime}\right\rangle=0.55$ (dash), 0.60 (solid) and 0.65 (dotted) from the laboratory frame ion signal (scatter) and its Legendre polynomial fit (red dashed-dotted line).

ionization profiles are found at the lowest intensity $(1.0 \times$ $10^{14} \mathrm{~W} \mathrm{~cm}^{-2}$ ) while more isotropic responses that could indicate saturation of single ionization are found at the highest intensity $\left(2.3 \times 10^{14} \mathrm{~W} \mathrm{~cm}^{-2}\right)$. The most striking difference between MF strong field ionization profiles in $\mathrm{N}_{2}$ and $\mathrm{CO}_{2}$ is the presence of a maximum peaked at $0^{\circ}$ in $\mathrm{N}_{2}$ and a local minimum at the same angle for $\mathrm{CO}_{2}$. In figure 4(a), the ionization maximizes close to $42^{\circ}$. This can be understood intuitively by considering the orbital structure of $\mathrm{N}_{2}, \mathrm{~N}_{2}^{+}, \mathrm{CO}_{2}$ and $\mathrm{CO}_{2}^{+}$given in table 1 . While the ground state of $\mathrm{N}_{2}$ and $\mathrm{CO}_{2}$ share the same $\Sigma_{g}^{+}$symmetry, ionization from the HOMO leads to $\tilde{X}^{2} \Sigma_{g}^{+}$in $\mathrm{N}_{2}^{+}$and to $\tilde{X}^{2} \Pi_{g}$ in $\mathrm{CO}_{2}^{+}$. A $\Sigma \rightarrow \Sigma+|\epsilon, k\rangle$ transition has typically larger dipole matrix elements along the internuclear axis $\left(\mathrm{N}_{2}\right)$, and $\Sigma \rightarrow \Pi+|\epsilon, k\rangle$ transitions $\left(\mathrm{CO}_{2}\right)$ have larger dipole matrix elements at some intermediate angle $\left(\approx 42^{\circ}\right)$ from the internuclear axis. Another way to state this is the Dyson orbital that is 'ionized' is $\Sigma_{g}$ for $\mathrm{N}_{2}$ and $\Pi_{u}$ for $\mathrm{CO}_{2}$ [40, 41].

These results are consistent with those reported by Pavičić et al [27]. For the case of $\mathrm{CO}_{2}$, the MF ionization probability $I(\theta)$ in Pavičić are considerably sharper than the present results in figure 4 . There are several possible causes of this difference. Pavičić used the lowest possible laser intensity
Table 1. Symmetry and energy of selected states of neutral and singly ionized $\mathrm{N}_{2}$ and $\mathrm{CO}_{2}$.

\begin{tabular}{lccc}
\hline Species & State & Configuration & $\epsilon(\mathrm{eV})$ \\
\hline $\mathrm{N}_{2}$ & $X^{1} \Sigma_{g}^{+}$ & $\left(1 \sigma_{g}\right)^{2}\left(1 \sigma_{u}\right)^{2}\left(2 \sigma_{g}\right)^{2}\left(2 \sigma_{u}\right)^{2}\left(1 \pi_{u}\right)^{4}\left(3 \sigma_{g}\right)^{2}$ & 0 \\
\hline & $\tilde{X}^{2} \Sigma_{g}^{+}$ & {$\left[X^{1} \Sigma_{g}^{+}\right]\left(3 \sigma_{g}\right)^{-1}$} & 15.58 \\
$\mathrm{~N}_{2}^{+}$ & $\tilde{A}^{2} \Pi_{u}$ & {$\left[X^{1} \Sigma_{g}^{+}\right]\left(1 \pi_{u}\right)^{-1}$} & 16.72 \\
& $\tilde{B}^{2} \Sigma_{u}^{+}$ & {$\left[X^{1} \Sigma_{g}^{+}\right]\left(2 \sigma_{u}\right)^{-1}$} & 18.74 \\
\hline $\mathrm{CO}_{2}$ & $X^{1} \Sigma_{g}^{+}$ & $\left(1 \sigma_{g}\right)^{2}\left(1 \sigma_{u}\right)^{2}\left(2 \sigma_{g}\right)^{2}\left(3 \sigma_{g}\right)^{2}\left(2 \sigma_{u}\right)^{2}\left(4 \sigma_{g}\right)^{2}$ & 0 \\
& & $\left(3 \sigma_{u}\right)^{2}\left(1 \pi_{u}\right)^{4}\left(1 \pi_{g}\right)^{4}$ & \\
\hline & & {$\left[X^{1} \Sigma_{g}^{+}\right]\left(1 \pi_{g}\right)^{-1}$} & 13.78 \\
$\mathrm{CO}_{2}^{+}$ & $\tilde{X}^{2} \Pi_{g}$ & {$\left[X^{1} \Sigma_{g}^{+}\right]\left(1 \pi_{u}\right)^{-1}$} & 17.31 \\
& $\tilde{A}^{2} \Pi_{u}$ & {$\left[X^{1} \Sigma_{g}^{+}\right]\left(3 \sigma_{u}\right)^{-1}$} & 18.07 \\
& $\tilde{B}^{2} \Sigma_{u}^{+}$ & {$\left[X^{1} \Sigma_{g}^{+}\right]\left(4 \sigma_{g}\right)^{-1}$} & 19.39 \\
& $\tilde{C}^{2} \Sigma_{g}^{+}$ & &
\end{tabular}

to ionize the $\mathrm{CO}_{2}$ molecules, and we can see from figure 4 that the angular distribution becomes less well defined with higher intensities. Pavičić directly measured the distribution function $P\left(\theta^{\prime}, \phi^{\prime}\right)$ by Coulomb-exploding the molecules. Higher charge states showed a narrower angular distribution, 

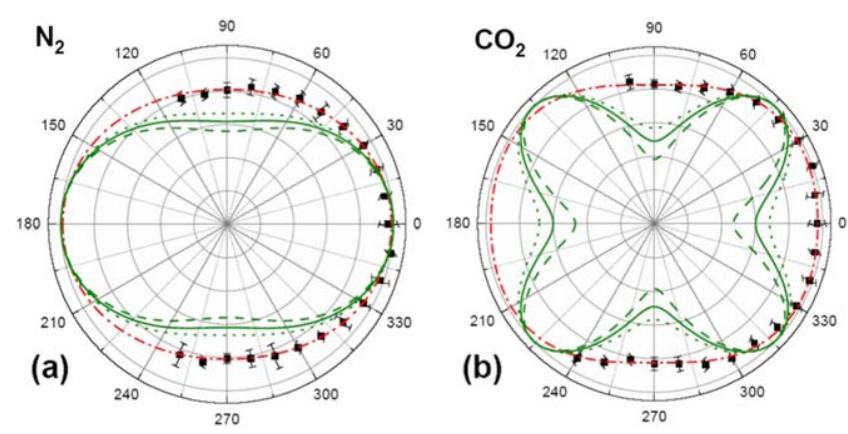

Figure 5. Strong field ionization versus alignment angle with $\lambda=1200 \mathrm{~nm}$ probe and intensity $1.1 \times 10^{14} \mathrm{~W} \mathrm{~cm}^{-2}$

(a) $\mathrm{N}_{2}$ (b) $\mathrm{CO}_{2}$. Legend is the same as in figures 3, 4 .

and so it may be that the direct measurement actually underestimated the degree of alignment. The fitting procedure then forced the molecular-frame ionization probability $I(\theta)$ to be narrower to match the laboratory-frame measurement.

To investigate the dependence of the MF ionization $I(\theta)$ on the wavelength of the ionizing laser, the OPA output at $1200 \mathrm{~nm}$ was used instead of $800 \mathrm{~nm}$. Figure 5 shows the measurements done with a probe wavelength of $1200 \mathrm{~nm}$ and intensity $1.1 \times 10^{14} \mathrm{~W} \mathrm{~cm}^{-2}$. The MF ionization profiles do not strongly depend on wavelength as profiles taken at $1200 \mathrm{~nm}$ (figure 5) are similar to those taken at $800 \mathrm{~nm}$ (figures 3 and 4). A clear comparison is difficult to make, as the laser intensities are difficult to determine exactly for the different wavelengths. The similarity between ionization at different wavelengths suggests that intermediate resonances in the multiphoton ladder leading to ionization do not play a major role in these molecules [42].

\subsection{High harmonic emission from aligned molecules}

The same experimental setup in the previous section, that measured the molecular-frame dependence of ionization yield, was used to record the molecular-frame dependence of HHG. As before, the polarization direction of the pump pulse that aligned the molecules in space was rotated by an angle $\alpha$; the probe pulse that generated the high-harmonic emission was always horizontal, so that the efficiency of the XUV spectrometer was the same. The polarization arrangement is shown in figure 6(a). The measurement covers the angular range $\alpha=-100^{\circ}$ to $100^{\circ}$ in steps of $5^{\circ}$, where each angular step is averaged over 1000 laser shots, and covers the spectral range of high-harmonic orders $H=17-41$. The HHG yields are plotted in polar coordinates, with the radial part corresponding to HHG photon energies from 0 to $80 \mathrm{eV}$. The emission occurs in discrete harmonics orders, but the color plots interpolate between them. The polar angle is $\alpha$, with $\alpha=0$ (horizontal) meaning that the molecular axis is parallel to the probe polarization axis that generated the HHG. The left and right halves of the polar plot are mirror images since there is no difference between the molecules at $0^{\circ}$ and $180^{\circ}$.

In figure 6(b), the HHG yield as function of angle is shown for $\mathrm{N}_{2}$ molecules probed with $800 \mathrm{~nm}$ laser pulses. The maximum emission occurs for $\alpha=0$, and minimizes at $\alpha=90^{\circ}$. This pattern is consistent with emission being primarily due to the $3 \sigma_{g}$ orbital of $\mathrm{N}_{2}$. A prominent minimum at
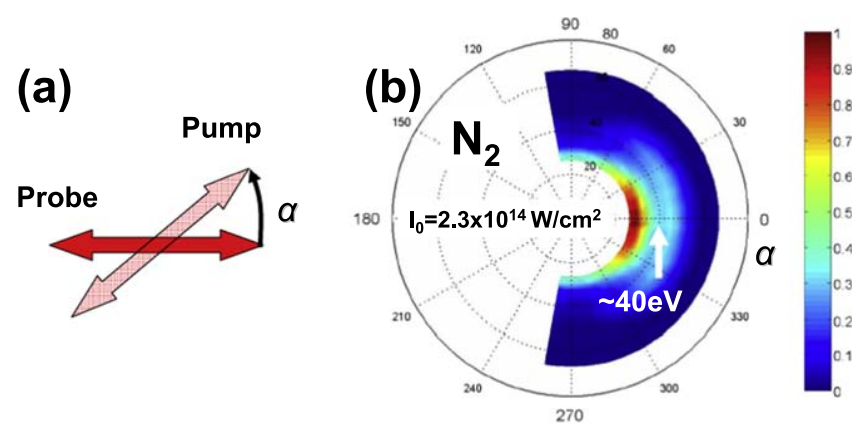

Figure 6. High-harmonic yield in aligned $\mathrm{N}_{2}$ at $\lambda=800 \mathrm{~nm}$ as a function of alignment angle $\alpha$. (a) Shows the polarization arrangement between pump and probe pulses. (b) Experimental intensity of $\mathrm{HHG}$ from aligned $\mathrm{N}_{2}$ molecules for a probe laser wavelength of $800 \mathrm{~nm}$. $I_{\text {probe }}=2.3 \times 10^{14} \mathrm{~W} \mathrm{~cm}^{-2} \cdot \alpha=0$ means that the molecular axis is parallel to the polarization of the pulse the generates the high-harmonic emission. The radial axis goes from 0 to $80 \mathrm{eV}$. The color scale is linear with intensity.

$40 \mathrm{eV}$ is observed as reported earlier [1, 15, 19, 26, 43, 44]. This minimum essentially remains present at all alignment angles $\alpha$ and is independent of laser parameters [19], and is attributed to a shape resonance in the photoionization cross section of $\mathrm{N}_{2}$. Another study reported a small spectral shift $(\sim 5 \mathrm{eV})$ of this minimum [45].

There has been evidence reported of the appearance of a structure near the cutoff at $\alpha=90^{\circ}$ that is associated with HOMO-1 [18, 26, 45, 46], but is not apparent here. To explain this, it is suggested that, in addition to the HOMO $(X X)$ and HOMO-1 $(A A)$ channels, an electron can crossrecombine from HOMO to HOMO-1 $(X A)$. RABBITT measurements [15] that led to the tomographic reconstruction of both HOMO and HOMO-1, suggest that the HOMO-1 channel is dominant at $\alpha=90^{\circ}$.

In figure 7, we present the high-harmonic yield of $\mathrm{CO}_{2}$ as a function of alignment angle $\alpha$ at driving field wavelength $\lambda=800 \mathrm{~nm}$ and three intensities. HHG spectra taken at $\alpha=0^{\circ}$ are presented in figure 8 for three peak intensities. We observe a spectral minimum which moves from $\mathrm{H} 25$ $(\sim 39 \mathrm{eV})$ to $\mathrm{H} 29(\sim 45 \mathrm{eV})$ on the intensity range covered. The intensity dependence of the position of this minimum was previously observed [7]. This feature is believed to be due to the interplay of multiple orbitals [14]. In aligned $\mathrm{CO}_{2}$, at $\alpha=0^{\circ}$, the spectral minimum is attributed to destructive interference between the HOMO (X) and HOMO-2 (B) channels. Additional experimental observation of this dynamical minimum at $\alpha=0^{\circ}$ and longer driving field wavelength was reported $(\lambda=1200 \mathrm{~nm} \mathrm{[19]} \mathrm{and} \lambda=1300 \mathrm{~nm}$ [47]) and supports the multiple orbitals interference model [14, 48-50].

Recent calculations [51] suggest that additional factors must be considered in the $\mathrm{HHG}$ from $\mathrm{CO}_{2}$. They show that electron-electron correlations and dipole interchannel couplings in the cation must be included. These calculations support the experimental observation in figure 7 that the spectral minimum at $\alpha=0^{\circ}$ goes to higher energy as the laser intensity is increased. The predicted minimum [51] of $42 \mathrm{eV}$ at $1.4 \times 10^{14} \mathrm{~W} \mathrm{~cm}^{-2}$ agrees exactly with our observation at $1.5 \times 10^{14} \mathrm{~W} \mathrm{~cm}^{-2}$ in figures $7(\mathrm{~b})$ and 8 . 

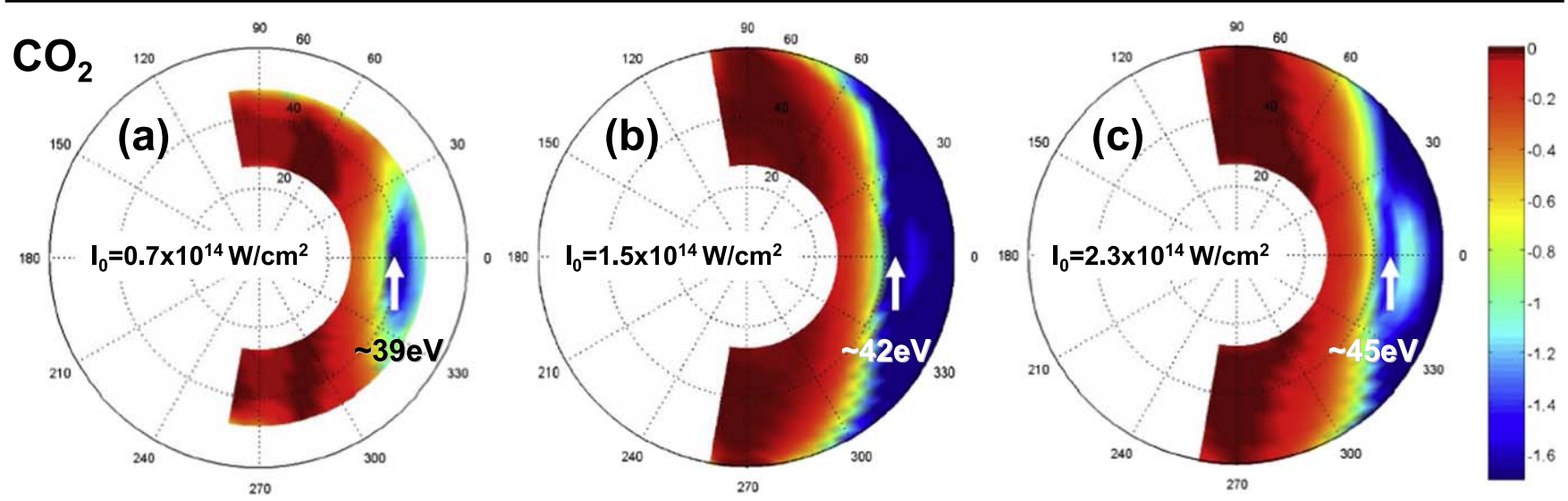

Figure 7. High-harmonic yield in aligned $\mathrm{CO}_{2}$ with $\lambda=800 \mathrm{~nm}$ as a function of alignment angle. Experimental measurements at $I_{\text {probe }}=0.7$ (a), 1.5 (b) and 2.3 (c) $\times 10^{14} \mathrm{~W} \mathrm{~cm}^{-2}$. The radial scale is $0-60 \mathrm{eV}$. The intensity scale is logarithmic $\left(\log _{10}\right)$. There is a characteristic minimum in the HHG spectrum for $\alpha=0$ that shifts with laser intensity.

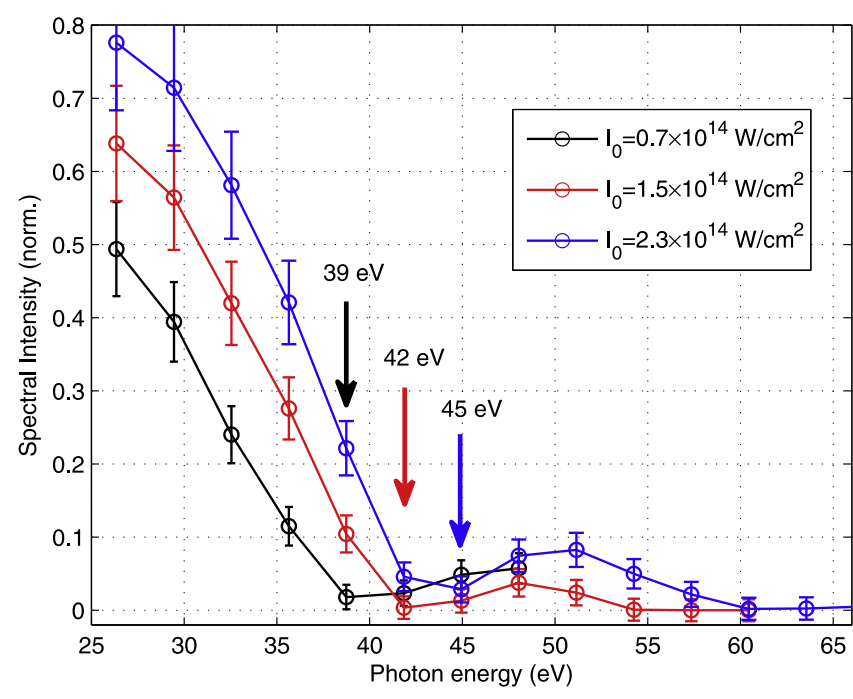

Figure 8. High-harmonic spectra in aligned $\mathrm{CO}_{2}$ for $\alpha=0^{\circ}$, i.e. molecular axis is parallel to the probe laser polarization. The probe wavelength is $\lambda=800 \mathrm{~nm}$. Experimental measurements at $I_{\text {probe }}=0.7$ (black solid line), 1.5 (red dashed line) and 2.3 (blue dotted line) $\times 10^{14} \mathrm{~W} \mathrm{~cm}^{-2}$. The minimum is seen to shift with increasing intensity to higher photon energy.

At low driving intensity $\left(I_{\text {probe }}=0.7 \times 10^{14} \mathrm{~W} \mathrm{~cm}^{-2}\right.$, see figure 7(a)), we observe that the high-harmonic yield of $\mathrm{CO}_{2}$ peaks around $\alpha \approx 60^{\circ}-70^{\circ}$ across the spectrum. This is consistent with EPOLYSCAT [52, 53] photorecombination transition dipole moments of the HOMO [54]. As the intensity increases, the angular profile peaks more around $\alpha=90^{\circ}$, see figures 7(b), (c) which suggests the dominance of the HOMO-1 participation at that angle as expected from both its corresponding angular ionization profile and transition moments peaking in that direction [14]. Finally, at the maximum intensity $\left(I_{\text {probe }}=2.3 \times 10^{14} \mathrm{~W} \mathrm{~cm}^{-2}\right.$, see figure $\left.7(\mathrm{c})\right)$, the local maximum of signal above the spectral minimum $(\geqslant 45 \mathrm{eV})$ in the vicinity of $\alpha=0^{\circ}$ has grown relatively to the overall yield, compared to $I_{\text {probe }}=1.5 \times 10^{14} \mathrm{~W} \mathrm{~cm}^{-2}$ (see figure 7 (b)), which roughly goes along with the increasing participation of HOMO-2. Therefore, we have shown that the angular high-

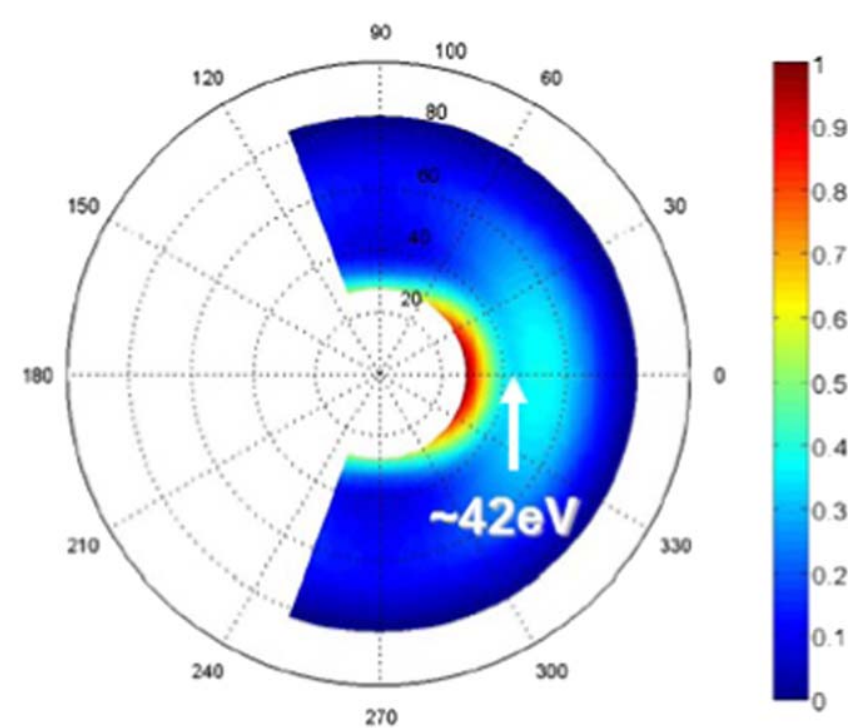

Figure 9. High-harmonic yield in aligned $\mathrm{N}_{2}$ versus $\alpha$ with probe driving wavelength $\lambda=1200 \mathrm{~nm}$. $I_{\text {probe }}=1.1 \times 10^{14} \mathrm{~W} \mathrm{~cm}{ }^{-2}$. The color scale is linear in HHG emission intensity. Compared with measurements taken at $800 \mathrm{~nm}$ (figure 6), the HHG spectrum has changed little at this wavelength.

harmonic yield profile depends strongly on intensity, giving insight into the participation of molecular orbitals.

HHG spectra of $\mathrm{N}_{2}$ taken with $1200 \mathrm{~nm}$ probe pulses are shown in figure 9. As discussed in [19], the position of the HHG minimum in $\mathrm{N}_{2}$ is fairly insensitive to laser parameters, as can be seen by direct comparison of figure 6(b) and of figure 9.

Figure 10 shows the $\mathrm{HHG}$ yield in $\mathrm{CO}_{2}$ for a probe laser wavelength of $1200 \mathrm{~nm}$. The minimum around $\alpha=0$ moves significantly towards higher photon energy with increasing intensity in the range $I_{\text {probe }}=1.2 \times 10^{14} \mathrm{~W} \mathrm{~cm}^{-2}$ [19]. It has been reported to reverse direction at higher intensity [47]. We have observed that when increasing the intensity to $I_{\text {probe }} \geqslant 1.5 \times 10^{14} \mathrm{~W} \mathrm{~cm}^{-2}$, the minimum remains around $58 \mathrm{eV}$ (not shown) and we have not seen the direction to reverse. 

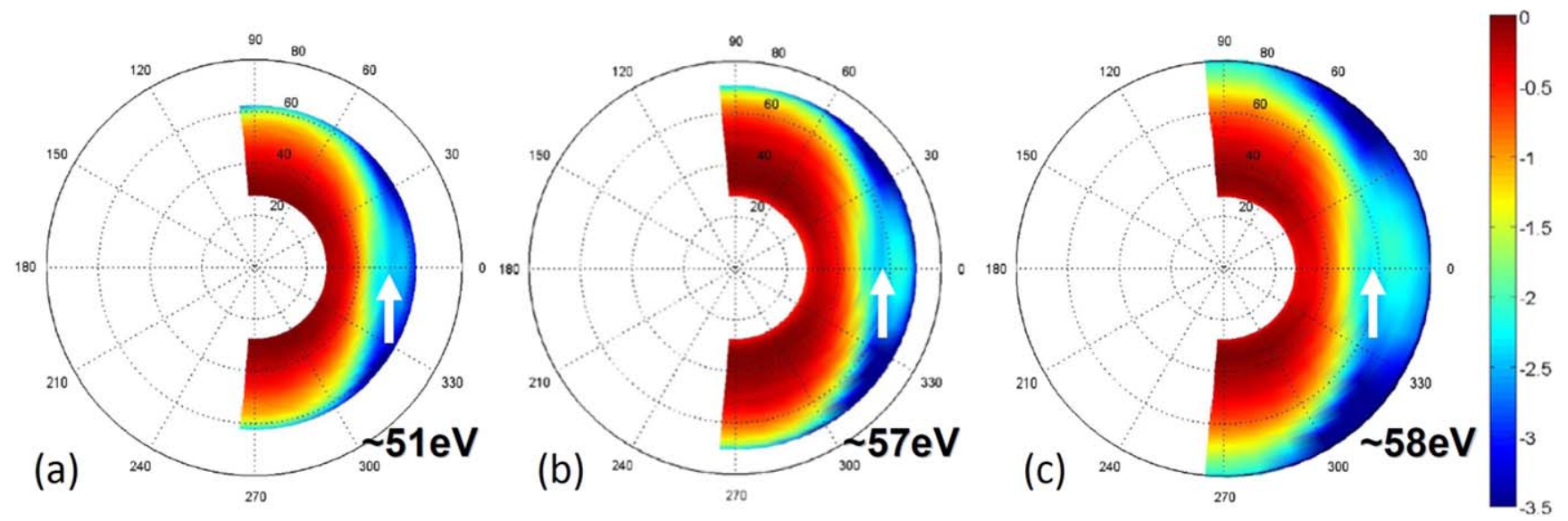

Figure 10. (a) High-harmonic yield in aligned $\mathrm{CO}_{2}$ as a function of alignment angle for $\lambda=1200 \mathrm{~nm}$ for three probe intensities. The radial axis goes from 0 to $80 \mathrm{eV}$ for all plots. The intensity scale is logarithmic $\left(\log _{10}\right)$.

\section{Conclusions and perspectives}

The behavior of the HHG spectra for $\mathrm{N}_{2}$ and $\mathrm{CO}_{2}$ around $\alpha=0$, i.e. when the laser polarization axis is parallel to the molecular axis, illustrates two contrasting mechanisms. The minimum in $\mathrm{N}_{2}$ around $40 \mathrm{eV}$ is largely independent of laser intensity and wavelength, and is an example of a structural minimum. The Cooper minimum in argon is an example of a structural minimum, whereby the photoionization cross section minimizes due to a sign change in one of the partial wave components [55].

On the other hand, the on-axis HHG minimum seen in $\mathrm{CO}_{2}$ is an example of a dyamical minimum, which is caused be the contribution of a deeper-bound orbital. In $\mathrm{CO}_{2}$ at $\alpha=0$, the contribution of the HOMO- 1 orbital is minimized, allowing the HOMO-2 to become apparent. Because the HOMO-2 has a higher ionization potential compared with the HOMO, it contributes to a higher cutoff energy in the HHG spectrum, where the HOMO contribution is falling. It is the coherent interference between HHG contributions from these orbitals that leads to the minimum in the HHG spectrum near the cutoff energy. As the laser intensity increases, so too does the location of the interference minimum. By going to longer laser wavelengths, the structural minimum of $\mathrm{CO}_{2}$ becomes apparent [56].

HHG has three steps: ionization, acceleration, and recombination. The simultaneous measurement of molecularframe ionization probability and the HHG yield will permit the deconvolution of the HHG yield from the laboratoryframe measurement to the MF [57], effectively removing the first step from the three-step model. This will improve the sensitivity of the measurement to the molecular-frame recombination matrix elements, and may provide more information on the molecular origins of HHG spectra.

\section{Acknowledgments}

We gratefully acknowledge support from the Joint Centre for Extreme Photonics.

\section{ORCID iDs}

C Marceau (iD https://orcid.org/0000-0002-8167-2727

H J Wörner (iD https://orcid.org/0000-0002-8877-0872

D M Villeneuve (ib https://orcid.org/0000-0002-2810-3648

\section{References}

[1] Itatani J, Levesque J, Zeidler D, Niikura H, Pépin H, Kieffer J C, Corkum P B and Villeneuve D M 2004 Nature 432867

[2] Haessler S et al 2010 Nat. Phys. 6200

[3] Vozzi C, Negro M, Calegari F, Sansone G, Nisoli M, De Silvestri S and Stagira S 2011 Nat. Phys. 7822

[4] Wörner H J, Bertrand J B, Kartashov D V, Corkum P B and Villeneuve D M 2010 Nature 466604

[5] Altucci C, Velotta R and Marangos J 2010 J. Mod. Opt. 57916

[6] Itatani J, Zeidler D, Levesque J, Spanner M, Villeneuve D M and Corkum P B 2005 Phys. Rev. Lett. 94 123902

[7] Kanai T, Minemoto S and Sakai H 2005 Nature 435470

[8] Wagner N L, Wüest A, Christov I P, Popmintchev T, Zhou X, Murnane M M and Kapteyn H C 2006 Proc. Natl Acad. Sci. 10313279

[9] Li W, Zhou X, Lock R, Patchkovskii S, Stolow A, Kapteyn H C and Murnane M M 2008 Science 3221207

[10] Baker S, Robinson J S, Haworth C A, Teng H, Smith R A, Chirila C C, Lein M, Tisch J W G and Marangos J P 2006 Science 312424

[11] Wörner H J, Bertrand J B, Corkum P B and Villeneuve D M 2010 Phys. Rev. Lett. 105103002

[12] Goulielmakis E et al 2010 Nature 466739

[13] Fleischer A, Wörner H J, Arissian L, Liu L R, Meckel M, Rippert A, Dörner R, Villeneuve D M, Corkum P B and Staudte A 2011 Phys. Rev. Lett. 107113003

[14] Smirnova O, Mairesse Y, Patchkovskii S, Dudovich N, Villeneuve D M, Corkum P B and Ivanov M Y 2009 Nature 460972

[15] Haessler S et al 2010 Nat. Phys. 6200

[16] Corkum P B 1993 Phys. Rev. Lett. 711994

[17] Corkum P B and Krausz F 2007 Nat. Phys. 3381

[18] McFarland B K, Farrell J P, Bucksbaum P H and Gühr M 2008 Science 3221232

[19] Wörner H J, Bertrand J B, Hockett P, Corkum P B and Villeneuve D M 2010 Phys. Rev. Lett. 104233904 
[20] Perelemov A M, Popov V S and Terent'ev M V 1966 Sov. Phys._JETP 23924

[21] Ammosov M V, Delone N B and Krainov V P 1986 JETP 641191

[22] Delone N B and Krainov V P 1998 Usp. Fizicheskikh Nauk. 41469

[23] Yudin G L and Ivanov M Y 2001 Phys. Rev. A 64013409

[24] Tong X M, Zhao Z X and Lin C D 2002 Phys. Rev. A 66 033402

[25] Otobe T, Yabana K and Iwata J-I 2004 Phys. Rev. A 69053404

[26] Mairesse Y et al 2010 Phy. Rev. Lett. 104213601

[27] Pavičić D, Lee K F, Rayner D M, Corkum P B and Villeneuve D M 2007 Phys. Rev. Lett. 98243001

[28] Rosca-Pruna F and Vrakking M J J 2001 Phys. Rev. Lett. 87 153902

[29] Stapelfeldt H and Seideman T 2003 Rev. Mod. Phys. 75543

[30] Salières P, L'Huillier A and Lewenstein M 1995 Phys. Rev. Lett. 743776

[31] Shiner A D, Trallero-Herrero C, Kajumba N, Bandulet H-C, Comtois D, Légaré F, Giguere M, Kieffer J-C, Corkum P B and Villeneuve D M 2009 Phys. Rev. Lett. 103 073902

[32] Dooley P W, Litvinyuk I V, Lee K F, Rayner D M, Spanner M, Villeneuve D M and Corkum P B 2003 Phys. Rev. A 68 023406

[33] Mairesse Y, Dudovich N, Zeidler D, Spanner M, Villeneuve D M and Corkum P B 2010 J. Phys. B: At. Mol. Opt. Phys. 43065401

[34] Yoshii K, Miyaji G and Miyazaki K 2009 Opt. Lett. 341651

[35] Lein M, Hay N, Velotta R, Marangos J and Knight P 2002 Phys. Rev. Lett. 88183903

[36] Petretti S, Vanne Y V, Saenz A, Castro A and Decleva P 2010 Phys. Rev. Lett. 104223001

[37] Petretti S, Magaña Á, Saenz A and Decleva P 2016 Phys. Rev. A 94053411

[38] Hoerner P, Lee M K and Schlegel H B 2019 J. Chem. Phys. 151054102
[39] Thomann I, Lock R, Sharma V, Gagnon E, Pratt S T, Kapteyn H C, Murnane M M and Li W 2008 J. Phys. Chem. A 1129382

[40] Patchkovskii S, Zhao Z, Brabec T and Villeneuve D M 2006 Phys. Rev. Lett. 97123003

[41] Patchkovskii S, Zhao Z, Brabec T and Villeneuve D M 2007 J. Chem. Phys. 126114306

[42] Spanner M and Patchkovskii S 2013 Chem. Phys. 41410

[43] Mairesse Y, Levesque J, Dudovich N, Corkum P B and Villeneuve D M 2008 J. Mod. Opt. 552591

[44] Schoun S B, Camper A, Salières P, Lucchese R R, Agostini P and DiMauro L F 2017 Phys. Rev. Lett. 118 033201

[45] Farrell J, McFarland B, Gühr M and Bucksbaum P 2009 Chem. Phys. 36615

[46] Tross J, Ren X, Makhija V, Mondal S, Kumarappan V and Trallero-Herrero C A 2017 Phys. Rev. A 95033419

[47] Torres R et al 2010 Phys. Rev. A 81051802

[48] Mairesse Y et al 2010 Phys. Rev. Lett. 104213601

[49] Le A-T, Lucchese R R, Lee M T and Lin C D 2009 Phys. Rev. Lett. 102203001

[50] Bertrand J B, Wörner H J, Salières P, Villeneuve D M and Corkum P B 2013 Nat. Phys. 9174

[51] Ruberti M, Decleva P and Averbukh V 2018 Phys. Chem. Chem. Phys. 208311

[52] Gianturco F A, Lucchese R R and Sanna N 1994 J. Chem. Phys. 1006464

[53] Natalense A P P and Lucchese R R 1999 J. Chem. Phys. 1115344

[54] Le A-T, Lucchese R R, Tonzani S, Morishita T and Lin C D 2009 Phys. Rev. A 80013401

[55] Wörner H J, Niikura H, Bertrand J B, Corkum P B and Villeneuve D M 2009 Phys. Rev. Lett. 102103901

[56] Vozzi C, Negro M, Calegari F, Sansone G, Nisoli M, Silvestri S D and Stagira S 2011 Nat. Phys. 7822

[57] Bertrand J B, Wörner H J, Hockett P, Villeneuve D M and Corkum P B 2012 Phys. Rev. Lett. 109143001 